\title{
Cosmological perturbations in transient phantom inflation scenarios
}

\author{
Martín G. Richarte ${ }^{1,2, a}$, Gilberto M. Kremer ${ }^{1, b}$ \\ ${ }^{1}$ Departamento de Física, Universidade Federal do Paraná, Caixa Postal 19044, Curitiba 81531-990, Brazil \\ ${ }^{2}$ Departamento de Física, Facultad de Ciencias Exactas y Naturales, Universidad de Buenos Aires, Ciudad Universitaria 1428, Pabellón I, \\ Buenos Aires, Argentina
}

Received: 9 December 2016 / Accepted: 15 January 2017 / Published online: 26 January 2017

(C) The Author(s) 2017. This article is published with open access at Springerlink.com

\begin{abstract}
We present a model of inflation where the inflaton is accommodated as a phantom field which exhibits an initial transient pole behavior and then decays into a quintessence field which is responsible for a radiation era. We must stress that the present unified model only deals with a single field and that the transition between the two eras is achieved in a smooth way, so the model does not suffer from the eternal inflation issue. We explore the conditions for the crossing of the phantom divide line within the inflationary era along with the structural stability of several critical points. We study the behavior of the phantom field within the slow-climb approximation along with the necessary conditions to have sufficient inflation. We also examine the model at the level of classical perturbations within the Newtonian gauge and determine the behavior of the gravitational potential, contrast density and perturbed field near the inflation stage and the subsequent radiation era.
\end{abstract}

\section{Introduction}

The standard inflationary paradigm is a theoretically appealing proposal invented to solve many problems which arise in the hot big bang model [1]. Although the latter one was extremely successful in explaining the observational evidence of an expanding universe, it had several problems which motivated the first inflationary models. For instance, the inflationary model was able to explain the flatness of the universe at large scale without using a fine-tuning mechanism to set the initial condition in the very early universe. The hot big bang suffers from the horizon problem, which means that different regions of the sky could not have been causally connected at the time of the hot big bang, how-

\footnotetext{
a e-mail: martin@df.uba.ar

be-mail: kremer@fisica.ufpr.br
}

ever, the almost uniform CMB temperature across the sky is a bullet proof of the large scale homogeneity in the universe [2]. Another issue that arises in the hot big bang model refers to the amount of monopoles observed in the universe. According to the grand unified theories of particle physics the hot big bang would produce a vast number of magnetic monopoles. The predicted length scales of their creation is such that the universe would be large enough at the GUT energy scale to contain a significant number, yet a magnetic monopole has never been observed so far in nature [3]. The inflationary scenario dictates that during some short epoch in the very early universe the non-zero vacuum energy density of some unknown field dominated the energy density of all other forms of energy, such as matter or radiation. In the simplest case, inflation is caused by a cosmological constant. A more complete scenario is inflation driven by a slowly rolling scalar field in a potential well. During the inflationary phase the scale factor of the universe grew exponentially so that initially small patches of space could have been stretched greater than the current observable universe [4]. One vital piece of this mechanism is the capability of explaining the origin of the primordial seeds for the cosmic large scale structure and fluctuations in the cosmic microwave background [5-7].

As was mentioned earlier, one of the most simplest scenario is a scalar inflaton field (associated with spin 0 particle) endowed with the property of having a sufficiently negative pressure to violate the strong energy condition. Moreover, the inflationary phase begins near the maximum of a flat potential; then the inflaton slowly rolls down the potential and finally the end of inflation is marked by oscillations at the minimum. This cornerstone of modern cosmology has been the subject of much investigation during the last decades [5].

There is a vast landscape of cosmological models where inflation can be achieved, for instance, vector model, extended gravity theory, gauge fields, coupled scalar fields, and many 
others [4-7]. However, we are going to focus our research on a recent proposal dubbed "phantom inflation" [8-13]. A somewhat natural manner to realize this scenario is by considering a self-interacting scalar field with a negative kinetic energy in the Lagrangian [14-19]. The reason for examining such a kind of inflation is threefold. First, phantom-like fields can be found in several types of well-accepted theories, for instance, k-essence models [20-25]. Besides, the current accelerating state of the universe can be properly described in terms of a phantom field with a negative equation state and this possibility cannot be discarded by the recent data extracted from Planck mission [26]. Third, the phantom inflation model has quite distinctive feature in relation with the standard model, which makes it an interesting alternative model. To be more precise, one peculiar fact is that the old minimal conditions to achieve inflation (the so-called slowroll approximation) must be replaced by a slow-climb mechanism provided the self-interacting potential must exhibit a maximum in order to avoid a fatal primordial big rip. Regarding the curvature power spectrum, the phantom inflation can also lead to a nearly scale invariant one, but its tensor perturbation is characterized by a blue tilt instead of a red one [8]. Nevertheless, one of the most critical shortcoming of the aforesaid model is the possibility of extending in time forever, thus not allowing for a graceful exit from the inflation era. This point was carefully analyzed first by Piao and Zhang [8]. These authors employed a hybrid extension of the phantom model by including an extra-normal scalar field, indeed, the coupling between them was designed for the phantom field to attain a slow-climb phase and then decay (for a certain critical mass scale) into radiation due to the presence of the normal scalar field [8]. The existence of a graceful exit from a phantom-like inflationary era was also explored in more detail in the context of non-minimally coupled gravity theory [27]; Feng et al. included an extra $\mathcal{F}(\mathcal{R}) \phi^{2}$ coupling in the phantom inflation model to achieve such a goal also, $\phi$ being the phantom field and $\mathcal{F}(\mathcal{R})$ a generic function of the Ricci scalar [27]. Interestingly enough, the new slow-climb conditions were derived and the general necessary conditions to avoid eternal phantom inflation were presented as well, finding that finite phantom inflation can be achieved without invoking new extra fields [27]. ${ }^{1}$

One of our aims in the present work is to show the possibility of having a phantom inflation era where the universe exhibits a pole-like behavior in the cosmic time and then transits to another matter phase without evoking new extra

\footnotetext{
${ }^{1}$ An issue that could be problematic is the quantum field theory associated with a phantom field. For instance, the model could lead to an instability of a vacuum state, however, one can always consider this field as an effective realization of a fundamental non-phantom theory well formulated in terms of the standard scheme of QFT. For a useful discussion of this point, the reader may see the references mentioned in [27].
}

fields. In doing so, the slow-climb conditions are satisfied during the short-inflation era. In order to achieve this goal, we are going to parametrize the scale factor with a double power law in terms of the cosmic time. With this proposal at hand, we will be able to reconstruct both the kinetic and the potential energy in terms of the cosmic time; in particular, we are going to obtain the shape of the potential in terms of the phantom scalar field in the limiting cases of large and small times. We examine the classical perturbation of this model, calculating the leading behavior of the gravitational Newtonian potential, the curvature perturbation along with the behavior of the perturbed phantom field. Such findings show that the extended model is free from the eternal inflation issue but more importantly the transition between the two eras is achieved in smooth way and without the inclusion of new-extra scalar field.

The paper is organized as follows. In Sect. 2, we present the phantom inflation model and discuss its main ingredients. In Sect. 3, the conditions for the crossing of the phantom divide line within the inflationary era are discussed along with the structural stability of several critical points. Section 4 is devoted to the analysis of the slow-climb approximation. In Sect. 5 the phenomenology of super-inflation is discussed. In Sect. 6 the analysis of the classical perturbation is performed. The conclusions are stated in Sect. 7.

\section{Transient phantom inflation}

Our starting point is to consider a phantom inflaton field minimally coupled to gravity. We then use as our action

$\mathcal{S}=\int \mathrm{d}^{4} x \sqrt{-g}\left[\frac{\mathcal{R}}{2}+\frac{1}{2} g^{\mu \nu} \partial_{\mu} \phi \partial_{\nu} \phi-V(\phi)\right]$,

where $\phi$ stands for the phantom (inflaton) field, $V$ represents the self-interacting potential and $\mathcal{R}$ is the Ricci scalar constructed from the metric theory. Throughout the article we work with natural (geometrical) units such that $M_{\mathrm{p}}^{2}=$ $8 \pi G=1$ and we adopt the metric signature $(-,+,+,+)$. Taking into account (1) we can derive the so called the Einstein field equations where the energy-momentum is of the form

$T_{\mu \nu}^{\phi}=-\frac{2}{\sqrt{-g}} \frac{\delta \mathcal{L}^{\phi}}{\delta g^{\mu \nu}}=-\mathcal{L}_{, X}^{\phi} \partial_{\mu} \phi \partial_{\nu} \phi+g_{\mu \nu} \mathcal{L}^{\phi}$.

Here $\mathcal{L}^{\phi}=X-V$ stands for the phantom Lagrangian (1), $X \equiv(1 / 2) g^{\mu \nu} \partial_{\mu} \phi \partial_{\nu} \phi<0$, and the suffix , $X$ represents a partial derivative with respect to $X$. Varying with respect to the phantom field it leads to a modified Klein-Gordon equation 
$\frac{1}{\sqrt{-g}} \partial_{\mu}\left(\sqrt{-g} \partial^{\mu} \phi\right)-V, \phi=0$.

We are going to work in the spatially flat FriedmannRobertson-Walker (FRW) metric with cosmic scale factor $a(t)$, namely, the metric is $g_{\mu \nu}=\operatorname{diag}\left[-1, a^{2}(t) \delta_{j}^{i}\right]$ with $t$ the cosmic time and $\delta_{j}^{i}$ the delta Kronecker tensor. The energy-momentum tensor of the phantom field (2) can be recast in terms of the one associated with a perfect fluid. Assuming an homogeneous phantom field, its pressure and energy density are given by

$\rho_{\phi}=-\frac{\dot{\phi}^{2}}{2}+V(\phi)$

$P_{\phi}=-\frac{\dot{\phi}^{2}}{2}-V(\phi)$,

where the dot stands for derivatives with respect to the conformal time. The Friedmann and the Raychaudhuri equations are given by

$3 H^{2}=\rho_{\phi}$,

$\frac{\ddot{a}}{a}=H^{2}+\dot{H}=-\frac{1}{6}\left(\rho_{\phi}+3 P_{\phi}\right)$,

where the Hubble parameter is defined as $H=\dot{a} / a$. Equation (7) tells us that an accelerated phase can be obtained if $\rho_{\phi}+3 P_{\phi}<0$, which is equivalent to $-2\left(\dot{\phi}^{2}+V\right)<0$. As one could expect, the weak energy condition is ruled out, that is, $\rho_{\phi}+P_{\phi}=-\dot{\phi}^{2}<0$. It should be remarked that the local energy conservation

$\dot{\rho}_{\phi}+3 H\left(\rho_{\phi}+P_{\phi}\right)=0$

is not altered within the phantom inflation provided it follows from Bianchi identity (i.e. $T_{; \mu}^{\mu \nu}=0$ ) regardless of the specific form of energy-momentum that appears in the r.h.s. of Einstein's equations. Moreover, Eq. (8) is equivalent to the modified KG:

$\ddot{\phi}+3 H \dot{\phi}-V_{, \phi}=0$.

At this point we are going to reconstruct the potential energy and the kinetic energy from a given scale factor so it is essential to show that procedure is easy to follow but also it will give us all the physical information encoded in the phantom inflationary scenario at the background level. Combining (6) with (8) we can obtain some formal expressions for the potential and kinetic term in terms of kinematic quantities:

$\dot{\phi}^{2}=2 \dot{H}$,

$V=\dot{H}+3 H^{2}$.

Knowledge of the evolution of $V$ and $\dot{\phi}^{2}$ requires one to solve the Friedmann equation (6) along with the conservation equation (8) consistently. In general, such a task is not easy provided the complexity and non-linearity of the above system of equations. In fact, the general exact solution for the scalar field is only known for an exponential potential [2832]. Our aim here is not to provide the general exact solution but to restrict our attention to a new (but quite general in some sense) kind of scale factor, which is an extended version of the power law. Given the scale factor $a(t)$, we will proceed to re-build the potential at the background level. Without further delays, we parametrize the scale factor as the product of two power laws:

$a(t)=a_{i} t^{\gamma}\left[1+\frac{t}{w}\right]^{\beta-\gamma}$,

where $a_{i}$ is a reference scale factor while $\beta, \gamma$, and $w$ are parameters of the double power-law model. Inflation will last for a period of time $t \in\left[0^{+}, t_{c}\right]$ and after $t>t_{c}$ the universe enters into non-accelerated phase. The physical motivation for this ansatz is connected with the string theory, where an exact solution with a pole behavior was reported [33,34]. Our purpose is to generalize this result within the framework of GR, giving simultaneously a new mechanism within the phantom scenario in which pole inflation decays toward a matter era at late times without introducing extra fields into the model. To make things clearer, we need to analyze the limiting behavior of the scale factor (12) for large and small values of the cosmic time. Let us first consider the case of a small cosmic time. Equation (12) becomes

$a \simeq a_{i} t^{\gamma}\left[1+\frac{t}{w}(\beta-\gamma)\right]$,

so the Hubble function is $H \simeq \gamma t^{-1}$ whereas the acceleration term approaches $\ddot{a} / a \simeq \gamma(\gamma-1) t^{-2}$. Bearing in mind the possibility of having an early accelerated era, we have two choices for $\gamma$; it could be $\gamma>1$ or $\gamma<0$. $^{2}$ Taking into account the behavior of $H$ and $\ddot{a} / a$, we can conclude that $\gamma>$ 1 is featured by accelerated contraction $(\dot{H}<0$ and $\ddot{a} / a>0)$ but the other case with $\gamma<0$ describes a phase of accelerated expansion driven by a phantom field with negative pressure. In fact, the aforesaid situation is more interesting provided the universe is super-accelerated because $\dot{H}>0$ and $\ddot{a} / a>0$, so the scalar curvature of the space-time is growing in the limit $t \simeq 0^{+}{ }^{3}$ Calculating the leading term in the energy density (6), we arrive at

\footnotetext{
2 We have dropped the $a_{0}$ factor for the sake of simplicity. However, they can be easily restored by re-scaling $a$ by $\frac{a}{a_{i}}$. The same kind of procedure can be done for the energy density, pressure, kinetic energy, and potential energy; for instance $p \rightarrow p / p_{i}, V \rightarrow V / V_{i}$, etc. We will consider the physical case in which $a_{i}>0, p_{i}>0, V_{i}>0$, and $\rho_{i}>0$.

3 The second scenario is characterized by $H<0, \dot{H}>0$, and $\frac{\ddot{a}}{a}>0$. Therefore, the Ricci curvature is defined positive provided $R=6[\dot{H}+$ $2 H^{2}$.
} 
$\rho_{\phi}(t)=3 \rho_{i} \gamma^{2} t^{-2}$.

Now, we give a physical interpretation of the dimensionless constant $w$. As is well known, during inflation, the scale energy is fixed by the inflaton. This equivalent to say that the energy during inflation should scale as $\rho_{\mathrm{inf}} \simeq \Lambda^{4}, \Lambda$ being a typical scale related with the Planck mass. Identifying the constant in (14) with the cutoff $\Lambda$, we obtain $\rho_{i}=\Lambda^{4} /\left(3 \gamma^{2}\right)$, $\rho_{i}$ being an initial energy scale; such value could be related with the critical density of the universe today. A better physical insight can be gained by calculating the leading term in the pressure given by $P_{\phi}=-\left[2 \dot{H}+3 H^{2}\right]$. It turns out that the pressure is given by

$P_{\phi}(t)=\rho_{i} \gamma(-3 \gamma+2) t^{-2}$.

Plugging (14) into (15), we see that the phantom field behaves as a dressed cosmological constant provided the new equation of state is $P_{\phi}=-\mu \rho_{\phi}$ with $\mu=(1+2 / 3|\gamma|)>0$ for $\gamma<0$. Equation (10) tells us that the leading term in the kinetic energy is $\dot{\phi}^{2}(t) \simeq-2 \gamma t^{-2}$; then the phantom field can be recast as $\phi=\phi_{0}-\epsilon_{ \pm} \sqrt{2|\gamma|} \ln t$ with $\epsilon_{ \pm}= \pm 1$. During this regime $\rho_{\phi} \simeq V$, so the potential can be written as

$V=V_{1} \exp \left[\lambda_{1} \epsilon_{ \pm}\left(\phi-\phi_{0}\right)\right]$

where $V_{1}=V_{i} \gamma(3 \gamma-1)$ and $\lambda_{1}=(2 /|\gamma|)^{1 / 2}$. An interesting fact is that the potential (16) can be the exponential one but also its counterpart; a negative exponential. Notice that the positivity of $V_{1}$ and the reality of $\lambda_{1}$ are both guaranteed provided the condition $\gamma<0$ holds for pole inflation (or super-inflation).

To this end, we need to explore the physical outcomes for considering a large cosmic time. The scale is given by

$a(t) \simeq a_{i} \frac{t^{\beta}}{w^{(\beta-\gamma)}}$.

From (17), we obtain $H=\beta t^{-1}$ along with $\ddot{a} / a \simeq \beta(\beta-$ 1) $t^{-2}$. A critical issue in phantom inflationary model is to ensure a graceful exit from that primordial era, that is, allowing a transition toward a non-accelerated era such as radiation or matter. Therefore, a non-accelerated regime after phantom inflation is achieved if $0<\beta<1$ provided $\ddot{a}<0$ and $\dot{H}=-\beta t^{-2}<0$. On the other hand, the density and pressure associated with the phantom fields are given by

$\rho_{\phi} \simeq 3 \rho_{i} \beta^{2} t^{-2}$,

$P_{\phi} \simeq p_{i} \beta(2-3 \beta) t^{-2}$.

Identifying the energy density (18) in terms of the scale factor $\rho_{\phi} \simeq 3 \rho_{i} \beta^{2} w^{-2(1+|\gamma| / \beta)}\left(a / a_{i}\right)^{-2 / \beta}$ with a radiation-like matter $\rho=\rho_{r}\left(a / a_{i}\right)^{-3 \gamma_{r}}\left(\gamma_{r}\right.$ being the barotropic index for radiation matter), one obtains $\rho_{r}=3 \beta^{2} \rho_{i}\left[w^{-2(1-\gamma / \beta)}\right]$, so $w=\left[3 \beta^{2}\left(\rho_{i} / \rho_{r}\right)\right]^{m}$ with $m=\beta / 2(\beta+|\gamma|)>0$. To have exact radiation $\beta$ should be fixed at the $1 / 2$ value. Furthermore, combining (18) and (19), we find that the equation of state is $P_{\phi} \simeq\left(\frac{1}{3}+\Delta\right) \rho_{\phi}$; so it is quite similar to radiation as long as $0<\beta<(2 / 3)$. One can estimate the deviation from a radiation matter encoded in the value of $|\Delta|=(2 / 3)|(1 / \beta)-2|$. The potential in terms of the cosmic time can be recast as $V \simeq V_{i} \beta(3 \beta-1) t^{-2}$. However, the identification by means of the phantom field is not sot evident because the phantom field becomes oscillatory, namely, $\phi=\phi_{0}+\epsilon_{ \pm} i \sqrt{2 \beta} \ln t$ and the potential is represented as $V \simeq V_{i} \beta(3 \beta-1) e^{i \epsilon_{ \pm} \lambda_{2}\left(\phi-\phi_{0}\right)}$ with $\lambda_{2}=\sqrt{2 / \beta}$ as well.

\section{Equation of state and the structural stability}

Let us begin this section by mentioning some important comments regarding the behavior of equation of state associated with the inflationary phantom model. As is well known, phantom inflation should have a graceful exit along with a crossing of the phantom divide and only then should exit the accelerated stage [41,42]. Some authors found that a k-essence associated with a scalar field with the quadratic kinetic term cannot achieve the crossing of the phantom divide in a physically plausible way $[41,42]$. So, we are going to see what happens with our model and the crossing of the phantom divide. To do so, we need to calculate the equation of state parameter defined as $\mathcal{W}(t)=-1-2 \dot{H} / 3 H^{2}$. In doing so, we use the scale factor (12) along with its derivatives to express the equation of state parameter in the following form:

$\mathcal{W}(t)=-1+\left(\frac{\beta t^{2}+2 \gamma w t+\gamma w^{2}}{\beta^{2} t^{2}+2 \gamma \beta w t+\gamma^{2} w^{2}}\right)$.

Equation (20) tells us that near the origin the value of the equation of state parameter does not depend on other parameters than $\gamma$, namely $\mathcal{W}\left(t \rightarrow 0^{+}\right)=-1+2 / 3 \gamma$. In order to have an initial inflationary phase one has to impose $\gamma<0$ and therefore $\mathcal{W}$ is clearly negative. In the opposite case, we obtain $\mathcal{W}(t \rightarrow \infty)=-1+2 / 3 \beta>0$ for $0<\beta<2 / 3$; once again the non-accelerated phase is controlled by the parameter $\beta$ only. For instance, one can have a matter dominated era by choosing $\beta=1 / 3$ or a radiation phase for $\beta=1 / 2$ and both cases satisfy the condition $\mathcal{W}(t \rightarrow \infty)>0$. From the latter results, it is clear that there is a smooth transition between an accelerated phase toward a non-accelerated final stage, which is one of the main purposes of the present work.

Another fact that can be noticed from Eq. (20) is that the crossing of the phantom divide, namely $\mathcal{W}\left(t_{c}\right)=-1$, can be achieved when $\dot{H}$ vanishes. The latter fact is equivalent to the requirement that the quadratic equation in Eq. (20) must have at least one zero. In order to gain some insights about that point, we are going to choose $w=1$ and $\gamma=-100$ 
for the rest of the discussion. Nevertheless, this choice is quite generic and we are not losing generality in the process of analyzing the crossing divide. We are going to consider two physical cases, the one with $\beta_{m}=1 / 3$ associated with a final era dominated by matter and another case with $\beta_{r}=1 / 2$ which corresponds to a radiation dominated era. For $\beta_{m}=1 / 3$, we see that the condition $\dot{H}=0$ is obtained twice, indicating that the universe crosses the phantom divide twice also. To be more precise, the numerical analysis shows that the condition $\mathcal{W}=-1$ is reached first at $t_{-}^{m} \simeq 55.01 \mathrm{seg}$ and later at $t_{+}^{m} \simeq 544.94 \mathrm{seg}$. Of course, one expects that the universe leaves the accelerated initial stage after having crossed the divide line so the time at which the acceleration is zero, called $t_{c}$, must be greater than $t_{+}^{m}$. In the $\beta_{m}=1 / 3$ case we find that $t_{c}=668.04 \mathrm{seg}>t_{+}^{m}$. The same situation happens for the case with $\beta_{r}=1 / 2$ in the sense that the universe crosses the phantom divide line twice and the last crossing occurs before the universe enters the non-accelerated phase. However, the universe dominated by radiation reaches the last crossing of the phantom divide faster than the universe dominated by matter. In order to really understand how our model does differ from previous proposals based on phantom models [41,42], we need to explain how the final stage can be achieved in terms of this "phantom" field and we will return to this point later.

We are going to investigate now the existence of asymptotic power-law solutions by means of a structural stability analysis, in other words, we are going to unveil the asymptotic nature of potentials allowing such solutions. To this end we introduce the equation of state parameter $\mathcal{W}=p / \rho$, and from the definitions above (4-5) and the Einstein equations (6) along with the conservation equation (8) one gets the dynamical equations for $\mathcal{W}(t)$, namely

$\dot{\mathcal{W}}=(\mathcal{W}-1)\left[\frac{\dot{V}}{V}+3 H(\mathcal{W}+1)\right]$.

In addition, one has to use the expression $-2 V=(\mathcal{W}-1) \rho$ along with Eq. (9) to obtain the above master equation (21). The reason for studying the stability of power-law solution in terms of the equation of state parameter is that the universe has a scale factor given by a power-law solution in the initial and final stages. Most importantly, the physical motivation for choosing a master equation with the $\mathcal{W}$ as a physical variable is that the universe has a constant equation of state parameter at the beginning of its evolution. Clearly, the solution $\mathcal{W}_{m}=1$ represents an equilibrium point, but it corresponds to non-accelerated expansion, specifically, $a \propto t^{2 / 3}$. Let us require then that Eq. (21) admits another equilibrium point with $\mathcal{W}=\mathcal{W}_{c}$, representing in consequence a solution with $a \propto t^{2 / 3\left(\mathcal{W}_{c}+1\right)}$. In particular, a solution representing a phantom early-time repeller would be characterized by a constant and negative value of $\mathcal{W}_{c}$. It is important to remark that contrary to what happens with a phantom field used to describe an era dominated by dark energy at late times [43], the initial inflationary stage associated with the critical point $\mathcal{W}_{c}$ must be a repeller; otherwise the universe cannot leave the aforesaid phase and the transition to another era would not be possible at all. Below, we will show that the requirement of the existence of such an equilibrium point restricts the functional form of the $V(\phi)$ potential. Then we formulate a structural stability analysis of the equation governing the evolution of the parameter $\mathcal{W}$. This is equivalent to imposing the asymptotic condition on the potential $\dot{V}+3(\mathcal{W}+1) H V=0$, which can be integrated to give $V=V_{0} a^{-3\left(\mathcal{W}_{c}+1\right)}$ with $V_{0}$ a positive integration constant. This finding as regards the form of the potential is completely consistent with our previous result (14) provided $\rho \simeq V(t)$. By choosing this potential the asymptotic regime of $\mathcal{W}$ is governed by the equation

$\dot{\mathcal{W}}=3 H(\mathcal{W}-1)\left[\mathcal{W}-\mathcal{W}_{c}\right]$

Let us now define $\mathcal{W}=\mathcal{W}_{c}+\epsilon$ with $0<\epsilon \ll 1$ and recast Eq. (22) in terms of this new definition. By expanding the r.h.s. of this equation up to order $\epsilon$, one obtains $\dot{\epsilon}=3 H \epsilon\left(\mathcal{W}_{c}-1\right)$. Then, for initially contracting universes $(H<0)$, the solution $\mathcal{W}=\mathcal{W}_{c}$ is a repeller provided $\mathcal{W}_{c}<0$, which is on the other hand our working hypothesis for having an inflationary stage $(\gamma<0)$. As has been shown, the initial stage must be associated with a repeller in order to have a transition to a second non-accelerated phase otherwise a graceful exit cannot be guaranteed. We are in a position to investigate the stability of the solution $\mathcal{W}_{c}\left(t_{b w}\right)=-1$ associated with the last transition of the phantom divide line. If we impose the fulfillment of Eq. (22) around $t_{b w}$ one obtains $\dot{V} / V=0$ with $V=V_{0}$ a constant. Now defining $\mathcal{W}=-1+\epsilon$ with $0<\epsilon<<1$ and using again (22) it follows that the master equation becomes $\dot{\epsilon}=3 H(\epsilon-2)^{2} \simeq 12 H(1-\epsilon)>0$. So for the inflationary phase with $H>0$ leads to an unstable transition at $\mathcal{W}_{c}=-1$, however, such a transition is harmless because the universe must cross the divide line before enters the non accelerated era. If the transition were characterized by an attractor point (stable) the phantom inflation would not be able to cross the phantom divide and therefore it would remain in that attractor forever, indicating that the model is not physically interesting provided cannot guaranteed the graceful exit from inflation along with the crossing of the phantom divide before moving forward the second stage $[41,42]$. At this point, it should be remarked that the analysis of instability associated with the crossing of the divide line performed in Ref. [43] does not hold in our case. The main reason is that in our inflationary stage the field is real and does not go like $\phi=t$, so their working hypotheses are different from the ones used in our work. Furthermore, we have shown that the structural stability of the power-law solution imposes 
the form of the potential and consistently the Friedman equation along with the Klein-Gordon equation determines the asymptotically shape of the phantom field. Nevertheless, one can consider both analyses as complementary ones given the fact that they describe different cosmological scenarios.

Let us end this section by showing that there is a symmetry in the proposed model or Lagrangian which can help us to understand the final stage where the model is accommodated as a scalar field which describes a radiation- or matter-like era. The Lagrangian is given by $\mathcal{L}=(1 / 2) \partial_{\mu} \phi \partial^{\mu} \phi-V(\phi)$. We begin by considering the existence of a symmetry which modifies the field in a global phase, namely $\phi \rightarrow i \varphi$ and $\partial^{\mu} \phi \rightarrow i \partial^{\mu} \varphi$, so the transformed Lagrangian becomes $\mathcal{L}^{\prime}=(1 / 2) i^{2} \partial_{\mu} \varphi \partial^{\mu} \varphi-V(i \varphi)$. Therefore, a realization of the symmetry only requires potentials which remain real under the transformation of the field $\phi \rightarrow i \varphi$ with $\varphi$ real. The existence of this kind of symmetry is equivalent to say that the original phantom field becomes a quintessence field with a normal kinetic energy. It is exactly because of the aforesaid property that the model can describe the initial era with a phantom Lagrangian and a final era with a quintessence Lagrangian. To be more precise, during the initial era it behaves as a phantom (real) field and then it becomes an pure imaginary field which leads to another scalar field with the normal kinetic energy and therefore it is suitable to account for a radiation era or a matter-like stage. One way to understand this point is with the help of Eq. (10), during the first accelerated era $\dot{H}>0$ so $\dot{\phi}^{2}$ remains positive and the model is associated with a phantom density, namely $\rho=-\left(\dot{\phi}^{2}\right) / 2+V(\phi)$. When the universe enters the non-accelerated phase, this means that $\dot{H}<0$ and by virtue of Eq. (10) it leads to $\dot{\phi}=i \sqrt{|2 \dot{H}|}=i \dot{\varphi}$ then the energy density becomes $\rho^{\prime}=+\left(\dot{\varphi}^{2}\right) / 2+V(\varphi)$. The previous statement is consistent with our findings reported at the end of Sect. 2, where we obtained $\varphi=\varphi_{0}+\epsilon_{ \pm} \sqrt{2 \beta} \ln t$ and the potential becomes real in terms of the $\varphi$ field, namely $V \simeq V_{i} \beta(3 \beta-1) e^{\epsilon_{ \pm} \lambda_{2}\left(\varphi-\varphi_{0}\right)}$.

\section{Slow-climb approximation}

The condition for having inflation is $\rho_{\phi}+3 P_{\phi}=-2\left(\dot{\phi}^{2}+\right.$ $V)<0$. The previous condition should be valid sufficiently long time in order to make the universe sufficiently flat. The standard approximation technique for analyzing inflation is the slow-roll approximation [4], however, this approach must be re-considered in the phantom scenario providing the negativity of kinetic energy term. In turns out that a viable phantom inflationary model must fulfill the following conditions, called slow-climb criteria $[8,13]$ :

- $|\dot{H}| \ll H^{2}$. This is equivalent to ensure $|\gamma| \gg 1$.

- $|\ddot{\phi}| \ll 3|H \dot{\phi}|$ or $3|\gamma| \gg 1$.

- $2 V \gg\left|-\dot{\phi}^{2}\right|$ or $3|\gamma| \gg 1$.
Taking into account the above results, we can roughly estimate the value of the slow-climb (SC) parameters in the primordial inflationary era. The SC parameters can be written as $[4,8,13]$

$\epsilon_{\mathrm{ph}}=-\frac{\dot{H}}{H^{2}}$,

$\delta_{\mathrm{ph}}=-\frac{\ddot{\phi}}{H \dot{\phi}}$,

and the difference between (23) and (24) is

$\eta_{\mathrm{ph}}=\left(\epsilon_{\mathrm{ph}}-\delta_{\mathrm{ph}}\right)$

Equations (23)-(26) tell us that $\epsilon_{\mathrm{ph}}=1 / \gamma, \delta_{\mathrm{ph}}=-1 / \gamma$ and therefore $\eta_{\mathrm{ph}}=2 / \gamma \neq 0$. Clearly, the condition $|\gamma| \gg 1$ implies the smallness of the slow-climb parameters. Additionally, similar results can be obtained when the slow-climb parameters are parametrized by the smallness of the potential slope and its concavity [4]. Then we arrive at $\epsilon_{\mathrm{Vph}}=(1 / 2)\left(V_{, \phi} / V\right)^{2}=1 /|\gamma| \ll 1$ along with $\eta_{\mathrm{Vph}}=V_{, \phi \phi} / V=2 /|\gamma| \ll 1$.

In a similar fashion, we can inspect the viability of the SC approximation by including higher terms of the hierarchy (cf. [4]). For instance, the square and cubic SC parameter are

$\xi_{\mathrm{Vph}}^{2}=\frac{V_{, \phi}}{V^{2}} V_{, \phi \phi \phi}$

$\sigma_{\mathrm{Vph}}^{3}=\frac{V_{, \phi}^{2}}{V^{3}} V_{, \phi \phi \phi \phi}$

Using Eqs. (26) and (27), we find $\xi_{\mathrm{Vph}}^{2}=(2 /|\gamma|)^{2}$ and $\sigma_{\mathrm{Vph}}^{3}=(2 /|\gamma|)^{3}$, respectively. For large $|\gamma|$, as one could expect, the following relations hold: $i-\sigma_{\mathrm{Vph}}^{3} \ll \xi_{\mathrm{Vph}}^{2} \ll$ $\eta_{\mathrm{Vph}}$ and $\mathrm{ii}-\sigma_{\mathrm{Vph}}^{3} \ll \xi_{\mathrm{Vph}}^{2} \ll \epsilon_{\mathrm{ph}}$. In the general case, this method leads to $\lambda^{n}=V_{, \phi}^{n-1} V_{, \phi}^{n+1} / V^{2}=(2 /|\gamma|)^{n}$, where the symbol $V_{, \phi}^{n+1}$ stands for the $(n+1)$-derivatives of $V$ with respect to its argument.

We are going to discuss some heuristic issues of our model. We begin by recalling that the effective spectral index is defined as [4]

$n_{s}(k)-1 \equiv \frac{\partial \ln \mathcal{P}_{s}}{\partial \ln k}$,

where $\mathcal{P}_{S}$ is the scalar power spectrum. In the case of powerlaw inflation with $\mathcal{P}_{S} \propto k^{n_{s}-1}$, the spectral index is a constant and is given by $n_{s}-1=-6 \epsilon_{\mathrm{Vph}}+2 \eta_{\mathrm{Vph}}=2 /|\gamma|$. Besides, the relative error in estimating $\mathcal{P}_{S}$ involves the error in $|\ln k|$, typically of order 0.01 and the error $\left|n_{s}-1\right|<0.03$ for the Planck mission [35]. The other magnitudes in calculating $\Delta \mathcal{P}_{S} / \mathcal{P}_{S}$ require an estimation of $n_{s}-1$ and $\mathrm{d} n_{s} / \mathrm{d} \ln k$ within the SC approximation. Such results can be extended from the book of Liddle and Lyth [4], in fact, neglecting higher corrections yields 
$n_{s}-1=2 /|\gamma|+\mathcal{O}\left(\xi_{\mathrm{Vph}}^{2}\right)$,

$\frac{\mathrm{d} n_{s}}{\mathrm{~d} \ln k}=-16 \epsilon_{\mathrm{Vph}} \eta_{\mathrm{Vph}}+24 \epsilon_{\mathrm{Vph}}^{2}+2 \xi_{\mathrm{Vph}}^{2}=\mathcal{O}\left(\sigma_{\mathrm{Vph}}^{3}\right)$.

Equation (29) tells us that to ensure some degree of accuracy of $n_{s}-1$ within the SC approach, $\left|\xi_{\mathrm{Vph}}^{2}\right| \ll$ $\max \left(\epsilon_{\mathrm{Vph}}, \eta_{\mathrm{Vph}}\right)=2 /|\gamma|$, whereas the slope of $n_{s}$ is zero within this approximation, so no error is introduced, as can be seen from (30).

We end this section by making some comments about the e-folding and its connection with the amount of inflation. A useful way to address the possibility of having super-inflation is by analyzing the number of e-folding. We need to examine if large values of $\gamma$ is consistent with having a sufficient time interval to inflation takes place. The amount of expansion during inflation is parametrized by the number of $e$-foldings $N$ which satisfies the following relation:

$\mathrm{d} N \equiv H \mathrm{~d} t=\mathrm{d} \ln a$.

From (31) and $\phi(t)$, we obtain $a \simeq a_{i} e^{-\lambda_{1} \epsilon_{ \pm}\left(\phi-\phi_{0}\right)}$. Taking $\epsilon_{ \pm}=+1$ and $\phi \simeq 0$ at the end of inflation, the amount of e-folding is $\Delta N \simeq \lambda_{1} \phi_{0}$. For $\Delta N>60$ the initial field is considerably small provided $\phi_{0}>\left(60 / \lambda_{1}\right) M_{p}$, where $M_{p}$ is the Planck mass and we restored the units for the sake of clarity. In another words, the end of inflation is identified with the breakdown of the slow-climb approximation. This is equivalent to $|\gamma| \simeq 1$ or $\epsilon_{\mathrm{ph}} \simeq 1$ and $\delta_{\mathrm{ph}} \simeq 1$. We can estimate the time $t_{\mathrm{f}}$ at which super-inflation ends by integrating (31) from an initial time (very close to zero) $t_{\mathrm{i}}$ to $t_{\mathrm{f}}$. It follows that $t_{\mathrm{f}}=t_{\mathrm{i}} e^{-\Delta N /|\gamma|}$ thanks to $H=-|\gamma| t^{-1}$. Starting from a Planck era characterized by $t_{\mathrm{i}}=t_{\text {Planck }} \simeq 10^{-43} \mathrm{~s}$, we get the standard value $t_{\mathrm{f}}=10^{-33} \mathrm{~s}$ by taking $|\gamma| \simeq \sqrt{7}$; this is an approximate value provided we are not considering the exact scale factor, however, the other corrections are sub-leading terms.

\section{The phenomenology of super-inflation with phantom fields}

Here we will collect and discuss basic mostly well-known facts about phantom cosmology, focusing on the mechanism of super-inflation. The main idea is to evaluate in further depth the phenomenology of the effective theory for a phantom cosmology and its implication for super-inflation. In particular, we will mention that a super-inflation model based on a phantom field still could be justified by considering it as an effective theory within a larger scheme. In doing so, we will mention several articles which deal with the perturbations of the phantom inflation in alternative frameworks.

The issue of phantom super-inflation was analyzed by several authors $[8-13,27]$. To be more specific, Piao et al. explored the issue of phantom inflation and the primordial perturbation spectrum in the case the universe exhibits a de Sitter phase [8]. In addition, they found that the issue of eternal inflation could problematic within this specific scheme and decided to include an additional normal scalar field, as is usually done in the context of the hybrid inflation model. In that paper, the authors already mentioned the possibilities of quantum instabilities when a phantom inflaton field is coupled to normal matter. Nevertheless, they considered that is much more important to focus in another aspect of the model as it may be the possibility of having a graceful exit from the inflationary stage toward a radiation era. Besides, the necessary conditions to prevent eternal inflation with the phantom field were examined by including stochastic effects in the Langevin equation [27], where a white Gaussian noise is responsible for the quantum fluctuations.

At this point, it is important to recall that our model does differ from the one reported in $[8,9,27]$ provided the background is completely different in several aspects. First, the scale factor is given by a double power-law function of the cosmic time. Second, the transition from an inflationary era toward a radiation phase is achieved within the phantom model without the inclusion of further degree of freedoms. Therefore, it is the phantom scalar field which decays into itself, converting the energy stored during inflation into radiation. Such aspects are not covered in Refs. [8,9,13,27]. Third, flipping the sign of slow-climb parameters associated with our model, we recover the usual result of the spectral index for the non-phantom field, showing the consistency of our results.

An alternative scenario to the standard inflationary model was examined by Piao et al.; they determined the behavior of primordial perturbation spectra with a phantom field but in the presence of expanding and contracting phase before the bounce [10]. Subsequently, Piao showed that the phantom field led to a very specific kind of primordial gravitational waves which can be used for looking new kind of physics. One of the important signature of the spin two perturbations associated with this model is the low amplitude for large scale and how it increases its magnitude at high frequencies [11].

We mention that our work is a clear extension of previous models based on phantom/ghost inflation cosmology and we are considering our model as a toy one. As pointed out by Piao in Ref. [12], the phantom scenario can be viewed as a phenomenological framework for describing a super-accelerated early stage of the universe. For example, phantom inflation can be accommodated in a class of warped compactification with the brane/flux annihilation within the context of string theory. The physical realization of this model showed the richness contained by the phantom super-inflation and why the model is should be considered as an effective one which can be embedded in a larger scheme, where the phantom 
fields are just one part of a fundamental theory with extra dimensions.

The rising of phantom behavior or the existence of ghost field appears in different context of scalar-tensor theory [37] or k-essence model [20-22,38]. So, our philosophy regarding the issue of the existence of phantom field is described in the following. The Lagrangian associated with the phantom field is assumed to be not an exact description of reality, but is rather an effective model which can be trusted at certain energy level or energy scale. As a matter of fact, it is widely known that the standard model of particle can only be trusted below an energy cutoff, which is near the Tev scale. Indeed, Carroll, Hoffman, and Trodden took the idea of an effective field theory as a point of departure for showing that a phantom model can be considered as a piece or a sector of a more fundamental theory [39]. Interestingly enough, the aforesaid authors demonstrated that there is a cutoff, close to the milli-electron volt, such that the phantom field would be stable over the lifetime of the universe [39]. In order to reinforce such an idea Arkani-Hamed et al. reported a ghost condensation within the context of an infrared modification of gravity which is consistent and compatible with all current experimental observations [40]. At this point, we must stress that in our model there is a transition from an initial (inflationary) era described by a phantom field and a final era described by a quintessence-like field (see Sect. 3 for further details).

\section{Classical perturbations}

Observations of the $\mathrm{CMB}$ reveal that the universe was nearly homogeneous at the time of decoupling. Small inhomogeneities only existed at the $10^{5}$ level which suggests that the conditions of the early universe may be accurately described by means of small perturbations on top of the homogeneous background solution. It is therefore natural to split quantities such as the metric, matter fields, and the stress tensor into a homogeneous background that depends only on cosmic time, and a small spatially dependent perturbation $[6,7]$.

We are interested in perturbations of the phantom field and the metric around the homogeneous background solution which we considered in the previous sections. To be more precise, we are going to consider $\phi(t, x) \rightarrow \phi(t)+\delta \phi(t, x)$, $g_{\mu \nu} \rightarrow g_{\mu \nu}+\delta g_{\mu \nu}$ along with the perturbed energymomentum $T_{\mu \nu} \rightarrow T_{\mu \nu}+\delta T_{\mu \nu}$. The dynamic of first order scalar perturbations of the metric tensor and the energymomentum tensor are coupled through the perturbed Einstein equation such that a perturbation in the energy-momentum density will induce a perturbation in the curvature of space time, $\delta G_{\mu \nu}=\delta T_{\mu \nu}$.

The metric for the background and scalar metric perturbations can be recast as $\mathrm{d} s^{2}=-(1+2 \Psi) \mathrm{d} t^{2}+(1-2 \Psi) a^{2}(t) \delta_{i j} \mathrm{~d} x^{i} \mathrm{~d} x^{j}$,

where $\Psi$ is the scalar mode of the perturbed metric. In fact, the form of the perturbed metric (32) indicates that the anisotropic tensor vanishes. $\Psi$ is called the curvature perturbation since it is the intrinsic scalar curvature of constant time hypersurfaces, namely, ${ }^{3} R=\left(4 / a^{2}\right) \nabla^{2} \Psi$. The specific form of (32) tells us that we are working within the Newtonian gauge. The components of the perturbed energy-momentum tensor for a scalar field read $[6,7]$

$\delta T_{0}^{0}=-\left[\Psi \dot{\phi}^{2}-\dot{\phi} \dot{\delta} \dot{\phi}+V_{, \phi} \delta \phi\right]$,

$\delta T_{j}^{i}=\left[\Psi \dot{\phi}^{2}-\dot{\phi} \dot{\delta} \dot{\phi}-V_{, \phi} \delta \phi\right] \delta_{j}^{i}$,

$\delta T_{i}^{0}=\frac{1}{a} \dot{\phi} \delta \phi_{, i}, \quad \delta T_{0}^{i}=-\frac{1}{a} \dot{\phi} \delta \phi_{, i}$,

where the dot stands for derivatives with respect to the cosmic time while the suffix , $i$ represents spatial derivatives [7]. Taking into account (32), (33), (34), and (35), the perturbed Einstein equations can be summarized

$3 H \dot{\Psi}+\left(3 H^{2}-\frac{\nabla^{2}}{a^{2}}\right) \Psi=-\frac{1}{2}\left[\Psi \dot{\phi}^{2}-\dot{\phi} \dot{\delta} \dot{\phi}+V_{, \phi} \delta \phi\right]$,

$(\dot{\Psi}+H \Psi)_{, i}=-\frac{1}{2} \dot{\phi} \delta \phi_{, i}$,

$\ddot{\Psi}+4 H \dot{\Psi}+\left(2 \dot{H}+3 H^{2}\right) \Psi=\frac{1}{2}\left[\Psi \dot{\phi}^{2}-\dot{\phi} \dot{\delta} \dot{\phi}-V_{, \phi} \delta \phi\right]$.

It is important to remark that the specific form of the perturbed Einstein equation along with the perturbed energymomentum for the phantom field is based on the metric signature adopted, so it will differ from the standard inflaton field equations of other references $[6,7] .{ }^{4}$ It is useful to recall that the curvature perturbation $\mathcal{R}$ is a gauge invariant quantity which in the Newtonian gauge can be written as $\mathcal{R}=\Psi+\mathcal{H} v^{N}$ [7], $v^{N}$ being the Newtonian velocity associated with the phantom field. Using (37) along with that analogy of a perfect fluid for the usual energy-momentum tensor [ i.e. $T_{i}^{0}=-\left(\rho_{\phi}+P_{\phi}\right) v_{, i}^{N}$ ] one can extract $v^{N}$ and

\footnotetext{
4 Nevertheless, our derivations are consistent with our previous choices made in the variational principle for obtaining the Einstein field equations. From our action (1) and applying the variational principle, we arrive at $G_{\mu \nu}=+T_{\mu \nu}$. Also, the specific form of the perturbed energy-momentum is consistent with the adopted Lagrangian for the phantom field, as one can easily check at the background level by using $L_{\phi}=X-V$ and the definition of $T_{\mu \nu}^{\phi}=-\frac{2}{\sqrt{-g}} \frac{\delta \mathcal{L}^{\phi}}{\delta g^{\mu \nu}}=$ $-\mathcal{L}_{, X}^{\phi} \partial_{\mu} \phi \partial_{\nu} \phi+g_{\mu \nu} \mathcal{L}^{\phi}$. In order to compare our results with previous work [7], some cautions must be taken in converting them and adapted to other frames. For instance, the energy-momentum of the perfect fluid is $T_{v}^{\mu}=(\rho+P) u^{\mu} u_{v}+P \delta_{v}^{\mu}$, while the constraint for the four-vector is given by $u^{\mu} u_{\mu}=-1$.
} 
consequently the curvature perturbation reads

$\mathcal{R}=\Psi+\frac{2}{3} \frac{\dot{\Psi}+H \Psi}{H(1+w)}$,

where we used that $P_{\phi}=w \rho_{\phi}$ along with the Friedmann equation $\left(3 H^{2}=\rho_{\phi}\right)$. In order to prove the constancy of this magnitude on superhorizon scale $(k \ll a H)$, we first obtain the dynamical equation for the gravitational potential at the classical level. To do so, we combine Eqs. (36)-(38) to get

$\ddot{\Psi}-\frac{\nabla^{2}}{a^{2}} \Psi+\dot{\Psi}\left(H-2 \frac{\ddot{\phi}}{\dot{\phi}}\right)+2 \Psi\left(\dot{H}-\frac{\ddot{\phi}}{\dot{\phi}} H\right)=0$.

With the help of the modified KG equation $\left(\ddot{\phi}+3 H \dot{\phi}-V_{, \phi}=\right.$ 0 ) along with the definition of adiabatic square speed for the phantom field $\left[c_{s}^{2}=\dot{P}_{\phi} / \dot{\rho}_{\phi}\right]$, one obtains

$c_{s}^{2}=\frac{-1}{3 H}\left(2 \frac{\ddot{\phi}}{\dot{\phi}}+3 H\right)$.

On the other hand, the equation of state $\left(w=P_{\phi} / \rho_{\phi}\right)$ satisfies the relation

$3(w+1) H^{2}=-2 \dot{H}$,

provided the well-known condition (10) holds. Replacing (41) and (42) into (40), the master equation for the gravitational field yields

$\ddot{\Psi}-\frac{\nabla^{2}}{a^{2}} \Psi+\dot{\Psi} H\left(4+3 c_{s}^{2}\right)+3 H^{2}\left(c_{s}^{2}-w\right) \Psi=0$.

We should mention that (43) can be used for showing the non-evolution of the curvature perturbation $\mathcal{R}$. Taking the derivative of (39), and employing (43), we obtain

$\dot{\mathcal{R}}_{k}=-\frac{k}{a H}\left[\frac{2 k \Psi_{k}}{3 a(1+w)}\right]$,

where we used a Fourier mode expansion for $\Psi=\Psi_{k}(t) e^{i k x}$ along with the relation $\dot{w}=-3 H(1+w)\left(c_{s}^{2}-w\right)$. Equation (44) tells us $\mathcal{R}_{k}$ remains constant on superhorizon scale for $k \ll a H$ if the bracket remains constant or vanishes in this limit also. The aforesaid fact is a crucial point because it enables us to connect perturbations generated during inflation to perturbations after the inflation even if we do not know anything about the physical mechanisms inside the horizon during the end of inflation and reheating [7]. In order to achieve such a goal, we expand the perturbed quantities in Fourier modes and use the background quantities such the scale factor, the phantom field, and the Hubble parameter given in
Sect. 2 in terms of the cosmic time. Taking into account the previous facts Eq. (40) or Eq. (43) becomes

$\ddot{\Psi}_{k}+\frac{(2-|\gamma|)}{t} \dot{\Psi}_{k}+\frac{\bar{k}^{2}}{t^{-2|\gamma|}} \Psi_{k}=0$.

Here $\bar{k}=k / a_{i}$. Equation (45) admits a closed solution in terms of a linear combination of the base $t^{q}\left\{J_{p}(n x), J_{-p}(n x)\right\}$ where $x=\left(\bar{k}^{1 /(1+|\gamma|)} t\right)^{(|\gamma|-1) / 2}, q=(|\gamma|-1) / 2<0$, $p=(|\gamma|-1) / 2(|\gamma|+1)>0$, and $n$ some constant which can be cast in terms of $|\gamma|$. We are not going to list that result here, however, there is a way to reduce (45) into the standard Bessel equation form by applying just a simple parametrization of the solution as $\Psi=t^{m} P(t)$ and choosing $m$ properly (see [36] for further details). We want to determine the form of the gravitational field when the cosmic time is very close to zero, thus we only need to capture the leading behavior of $\Psi_{k}$. Implementing the previous approach into Eq. (45), the gravitational field reads

$\Psi_{k}(t)=C_{1 k} t^{\frac{(1-|\gamma|)}{2}}+C_{2 k} t^{\frac{|\gamma|-1}{2}}$

where $C_{1 k}$ and $C_{2 k}$ are integration constants. It is clear from (46) that the gravitational field has two kinds of modes, a growing one and a decaying mode. Let us explore the physical consequences of coming from the leading term in Eq. (46). In the case of small $k /(a H) \propto\left(k / a_{i}\right) t^{(1+|\gamma|)}$, the derivative of the curvature perturbation (44) tells us that

$\dot{\mathcal{R}}_{k} \simeq-\frac{2 k^{2}}{3|\gamma|(1+w) a_{i}^{2}} t^{3 \frac{(1+|\gamma|)}{2}} \rightarrow 0$.

Bearing in mind all this information we need to find the behavior of the perturbed phantom field during inflation. One way to obtain $\delta \phi_{k}$ is by looking more closely to the perturbed Einstein constraint (36). It is important to note that the evolution of background quantities (namely, $\phi, V_{, \phi}$ and $H$ ) and the gravitational field $\Psi_{k}$ are known at this stage. Therefore, the perturbed Einstein constraint can be seen as a master equation for $\delta \phi_{k}$. Of course, all the physical results that we can extract from this equation are equivalent to those obtained from (37) or (38). We present (36) as a differential equation for $\delta \phi_{k}$

$\dot{\delta} \phi_{k}-\frac{\dot{V}}{\dot{\phi}^{2}} \delta \phi_{k}=S_{k}(t)$,

and the source term is given by

$S_{k}(t)=\frac{\Psi_{k}}{\dot{\phi}}\left(\frac{2 k^{2}}{a^{2}}+\dot{\phi}^{2}\right)+\frac{6 H^{2}}{\dot{\phi}} \Psi_{k}+\frac{6 H}{\dot{\phi}} \dot{\Psi}_{k}$.

Before solving (48), it is illustrative to determine the leading contribution of each term in (49). To be more precise, we 
obtain $\Psi_{k} / a^{2} \dot{\phi} \propto t^{3(1+|\gamma|) / 2}, \Psi_{k} \dot{\phi} \propto t^{-(1+|\gamma|) / 2}, \dot{\Psi}_{k} H / \dot{\phi} \propto$ $t^{-(1+|\gamma|) / 2}$, and $\Psi_{k} H^{2} / \dot{\phi} \propto t^{-(1+|\gamma|) / 2}$. In short, the leading contribution in (49) can be written as $S_{k}(t)=S_{0} t^{-(1+|\gamma|) / 2}$ with $S_{0}$ a constant. Replacing the latter result along with $\dot{V} / \dot{\phi}^{2}=-(3|\gamma|+1) t^{-1}$ in (48), we see that the perturbed field is

$\delta \phi_{k}(t)=D_{1 k} t^{-(3|\gamma|+1)}-\frac{2 S_{0}}{(7+5|\gamma|)} t^{(1-|\gamma|) / 2}$.

Both terms in Eq. (50) are growing modes in the limit of vanishing cosmic time when phantom inflation happens. However, the leading contribution is due to the homogeneous solution of (48). Equation (50) shows us that the perturbed (phantom) field grows without limit near inflation but it then decays at late times. Another physical quantity that we must calculate is the contrast density, at least its leading contribution. The evolution of $\delta \rho_{\phi}$ can be obtained from (33) using $\delta T_{0}^{0}=-\delta \rho_{\phi}$ along with (46) plus (50). It turns out that $\delta \rho_{\phi}$ has three contributions, namely, $\Psi \dot{\phi}^{2} \propto t^{-(3+|\gamma|) / 2}$, $\dot{\phi} \dot{\delta \phi} \propto t^{-(3|\gamma|+3)}$, and $V_{, \phi} \delta \phi \propto t^{-3(1+|\gamma|)}$. From the latter fact and the background energy density (14), we find that the density contrast behaves as

$\frac{\delta \rho_{\phi}}{\rho_{\phi}} \simeq \frac{\delta \rho_{i}}{3 \gamma^{2} \rho_{i}} t^{-3(|\gamma|+1)}$,

where $\delta \rho_{i}$ is an initial density contrast. As one might expect, Eq. (51) tells us that the density contrast grows like the perturbed phantom field, thus $\delta \phi_{k}(t) \propto t^{-3(|\gamma|+1)}$.

Of course, the analysis is not complete and it is important to establish what happens in the opposite limit, during a matter-like era. Following the same approach for examining the classical perturbation during a matter(radiation)-like era (the precise kind of era is controlling by the value of $\beta$ ), we obtain similar equations for the gravitational potential and therefore we are not going to repeat the procedure here. We find that the growing mode of gravitational field is $\Psi_{k} \propto t^{(\beta+1) / 2}$. We have two different types of behaviors for the perturbed field depending on the values which are taken by $\beta$. For $\beta \in(0,7 / 9)$, we find that the leading term in the perturbed field is $\propto t^{(-3 \beta+5) / 2}$ and density contrast is given by $\left(\delta \rho_{\phi} / \rho_{\phi}\right) \propto t^{(-3 \beta+5) / 2}$ also; the growing behavior of the density contrast at late time ensures the formation of structures. For $7 / 9<\beta<1$, the matter grows at a different rate, namely $\left(\delta \rho_{\phi} / \rho_{\phi}\right) \propto \delta \phi_{k}(t) \propto t^{(3 \beta-1)}$.

\section{Conclusions}

In this paper we have analyzed one transient model within the context of "phantom" inflation. We presented an exact solution where the scale factor interpolates between a pole-like inflation era and a radiation era at late times. Given a scale factor, we have reconstructed the potential energy and kinetic in terms of the cosmic time, further we found that the potential corresponds to an exponential one. An interesting fact of this model is that the phantom inflaton decays into a quintessence field converting the stored energy during inflation into coherent radiation without the need of an extra field to produce such mechanics. Besides, we showed that the phantom model is free of the eternal inflation issue. We examined the issue of a phantom inflationary model that crosses the divide line before the universe enters the non-accelerated second stage. We also showed that the original phantom Lagrangian exhibited a global symmetry which allowed us to explained the reason why the model could describe the radiation (or matter-like) final era.

We have examined the slow-climb approximation and determined the conditions under which the model can be considered as a viable inflationary scenario. The analysis was carried out with the help of the (kinematic) slow-climb parameters, showing that the parameter of the model must be considerable. We also went in the analysis beyond the slowclimb approximation by including the slow-climb parameters in terms of the potential. In that case, we calculated the effective spectral index and the running of it. Later, we analyzed the necessary conditions to have a sufficient amount of inflation.

We pointed out the difference among our model and other interesting proposals. The background dynamic is characterized by a scale factor which has a double power-law function of the cosmic time. The aforesaid trait allows the transition from an inflationary era toward a radiation phase within the phantom model without the inclusion of further degree of freedoms; such aspects are not covered in Refs. $[8,9]$.

At the level of classical perturbations we have obtained the growing and decaying mode of the gravitational field within the Newtonian gauge. We also have verified that the derivative of the curvature perturbation goes to zero for small $\mathrm{k} / \mathrm{a} H$. We have calculated the leading behavior of perturbed field during inflation; it turns out that the perturbed field grows like the contrast density and both behave as a power law (pole) with the cosmic time during inflation and then decays to zero at late times. In addition, the perturbed field and the contrast density both grow at late time.

Summarizing, unlike some previous work on phantom inflation (cf. [8]) where an extra scalar field is introduced to force the phantom field to decay, our model only has one phantom inflaton that decays into another quintessence field, such a field being responsible for both the inflationary epoch and the radiation era.

Acknowledgements We are grateful to Prof. Dr. A. Vikman for many useful comments on the article and the reviewer for some important 
suggestions. M. G. R. and G. M. K. are supported by Conselho Nacional de Desenvolvimento Científico e Tecnológico (CNPq)- Brazil.

Open Access This article is distributed under the terms of the Creative Commons Attribution 4.0 International License (http://creativecomm ons.org/licenses/by/4.0/), which permits unrestricted use, distribution, and reproduction in any medium, provided you give appropriate credit to the original author(s) and the source, provide a link to the Creative Commons license, and indicate if changes were made. Funded by SCOAP ${ }^{3}$.

\section{References}

1. A.H. Guth, Phys. Rev. D 23, 347356 (1981)

2. A.D. Linde, Phys. Lett. B 108, 389-393 (1982)

3. A. Albrecht, P.J. Steinhardt, Phys. Rev. Lett. 48, 12201223 (1982)

4. A.R. Liddle, D.H. Lyth, Cosmological Inflation and Large-Scale Structure (Cambridge University Press, Cambridge, 2000)

5. D. Lyth, A. Liddle, The Primordial Density Perturbation: Cosmology. Inflation and the Origin of Structure (Cambridge University Press, Cambridge, 2009)

6. V. Mukhanov, Physical Foundations of Cosmology (Cambridge University Press, Cambridge, 2005)

7. V.F. Mukhanov, H.A. Feldman, R. Brandenberger, Theory of cosmological perturbations. Phys. Rep. 215, 203333 (1992)

8. Y.S. Piao, Y.Z. Zhang, Phys. Rev. D 70, 063513 (2004)

9. Y.S. Piao, E. Zhou, Phys. Rev. D 68, 083515 (2003)

10. Y.S. Piao, Y.Z. Zhang, Phys. Rev. D 70, 043516 (2004)

11. Y.S. Piao, Phys. Rev. D 73, 047302 (2006)

12. Y.S. Piao, Phys. Rev. D 78, 023518 (2008)

13. J.E. Lidsey, Phys. Rev. D 70, 041302 (2004)

14. P.F. Gonzalez-Diaz, J.A. Jimenez-Madrid, Phys. Lett. B 596, 16 (2004). arXiv:hep-th/0406261

15. P.F. Gonzalez-Diaz, Phys. Rev. D 68, 084016 (2003)

16. S. Nojiri, S.D. Odintsov, Gen. Relativ. Grav. 38, 1285 (2006)

17. S. Capozziello, S. Nojiri, S.D. Odintsov, Phys. Lett. B 632, 597 (2006)

18. E. Elizalde, S. Nojiri, S.D. Odintsov, D. Saez-Gomez, V. Faraoni, Phys. Rev. D 77, 106005 (2008)
19. P. Wu, H.W. Yu, JCAP 0605, 008 (2006)

20. C. Armendariz-Picon, T. Damour, V.F. Mukhanov, Phys. Lett. B 458, 209 (1999)

21. T. Chiba, T. Okabe, M. Yamaguchi, Phys. Rev. D 62, 023511 (2000)

22. P.F. Gonzalez-Diaz, Phys. Lett. B 586, 1 (2004)

23. L.P. Chimento, R. Lazkoz, Phys. Rev. Lett. 91, 211301 (2003)

24. J.G. Hao, X.Z. Li, Phys. Lett. B 606, 7 (2005)

25. L.P. Chimento, Phys. Rev. D 69, 123517 (2004)

26. P. A. R. Ade et al., arXiv:1303.5076v1

27. C.J. Feng, X.Z. Li, E.N. Saridakis, Phys. Rev. D 82, 023526 (2010). arXiv: 1004.1874

28. L.P. Chimento, J. Math. Phys. 38, 2565 (1997)

29. L.P. Chimento, A.E. Cossarini, N.A. Zuccala, Class. Quantum Grav. 15, 17 (1998)

30. L.P. Chimento, Class. Quantum Grav. 15, 965 (1998)

31. L.P. Chimento, M.I. Forte, R. Lazkoz, M.G. Richarte, Phys. Rev. D 79, 043502 (2009)

32. L.P. Chimento, R. Lazkoz, M.G. Richarte, Phys. Rev. D 83, 063505 (2011)

33. M. Gasperini, G. Veneziano, Astropart. Phys. 1, 317 (1993)

34. M. Gasperini, arXiv:gr-qc/9706037

35. P. A. R. Ade et al., Planck Collaboration (2015). arXiv:1502.02114

36. L.P. Chimento, M.G. Richarte, Phys. Rev. D 93, 043524 (2016)

37. B. Boisseau, G. Esposito-Farese, D. Polarski, A.A. Starobinsky, Phys. Rev. Lett. 85, 2236 (2000)

38. T. Damour, V. Mukhanov, Phys. Lett. B 458, 209 (1999)

39. S.M. Carroll, M. Hoffman, M. Trodden, Phys. Rev. D 68, 023509 (2003)

40. N. Arkani-Hamed, H.C. Cheng, M.A. Luty, S. Mukohyama, JHEP 05, 074 (2004)

41. A. Vikman, Phys. Rev. D 71, 023515 (2005). arXiv:astro-ph/0407107

42. D.A. Easson, A. Vikman, arXiv: 1607.00996

43. Y. Ito et al., arXiv:1111.5389 